\title{
There is no unmet requirement of optical coherence for continuous-variable quantum teleportation
}

\author{
H.M. Wiseman* \\ Centre for Quantum Dynamics, School of Science, \\ Griffith University, Brisbane, Queensland 4111, Australia
}

February 1, 2008

\begin{abstract}
It has been argued [T. Rudolph and B.C. Sanders, Phys. Rev. Lett. $\mathbf{8 7}, 077903$ (2001)] that continuous-variable quantum teleportation at optical frequencies has not been achieved because the source used (a laser) was not 'truly coherent'. Here I show that 'true coherence' is always illusory, as the concept of absolute time on a scale beyond direct human experience is meaningless. A laser is as good a clock as any other, even in principle, and this objection to teleportation experiments is baseless.
\end{abstract}

Recently, Rudolph and Sanders (RS) published a letter entitled 'Requirement of Optical Coherence for Continuous-Variable Quantum Teleportation' [1]. In it they argued that, contrary to Ref. [2], continuous-variable quantum teleportation (CVQT) has not been and, in fact, cannot be, achieved using a laser as a source of coherent radiation. They base their argument on their claim that a laser is not a source of coherent radiation, in the sense that the output of a laser is not a coherent state, but an equal mixture of coherent states with all possible phases. As they correctly point out, following Mølmer [3], this can also be interpreted as a Poissonian mixture of number states. Thus, they argue, the description in Ref. [2] is invalid because it relies upon the 'partition ensemble fallacy' [- That is, its analysis is carried using one partition of the ensemble (into coherent states) because it would not be valid in another partition (into number states). Although the mathematics of RS is indisputable, their letter suffers from a deep conceptual problem.

For the argument of RS to have any teeth, they must allow that the production of coherent states of light is possible in principle, by some means other than a laser. Indeed they say that 'We therefore assert that genuine CVQT requires coherent devices, that is, devices capable of generating true coherence, and these are not a feature of current CVQT experiments.' If this were true 
then RS would have some basis for criticising at least the formalism of Ref. [2]. It is the point of this letter to show that even in principle there are no devices that can generate 'true coherence' any better than a laser. Moreover, if one insists on rejecting a laser as a coherent source, then one must discard much else besides.

Again emphasising that their argument relies upon the possible existence of 'true coherence', RS say that they 'are of course not asserting that production of coherent states of light is impossible: basic quantum electrodynamics shows that a classical oscillating current can produce coherent states.' The first problem with this claim is that, as far as we know, the universe is quantal, not classical. There is no reason for believing in classical electrical currents. Nevertheless, a suitable quantum current would generate a coherent state of light to an arbitrarily good approximation, so I will not belabour this point.

A more serious problem is that it is not possible to produce even such a quantum current, in a sense that would pass the 'truth' test of RS. The natural oscillators at optical frequencies are the electrons in atoms. Without coherent light, we could still make the atom 'ring' by 'striking' it (with a free electron, for example). However, for this oscillation to have a definite phase the time of the collision would have to be known to an accuracy less than an optical cycle, of order $10^{-15} \mathrm{~s}$. Otherwise one would have to average over all possible phases and one would be left with exactly the same problem as with the laser.

Achieving this accuracy would require a clock which ticks faster than $10^{15} \mathrm{~s}^{-1}$. Presumably this is the sort of 'technical challenge' that RS mention in the context of producing coherence by making a measurement on the gain medium of a laser. But it is much more than just a technical challenge. Instead, it just moves the problem back another step. How could we be sure that the clock has a definite phase? Perhaps it is fixed relative to another clock, but then we can ask the question again of that second clock. And so ad infinitum.

It is thus clear that the 'true coherence' as meant by RS is impossible to produce technologically. It cannot mean coherent relative to any clock, because their argument attacking the phase of a laser can be equally used to attack that of any clock. Therefore, if it means anything, 'true coherence' must mean coherent relative to an absolute time standard for the universe. Since such a hypothetical absolute time standard can never be measured, I would maintain that it is meaningless, and with it the idea of 'true coherence' in the sense of RS.

It might be countered that as conscious beings we experience the flow of absolute time directly, and so give it meaning. Admitting the validity of this temporal experience (which does not go without saying [5]), this argument nevertheless cannot work to establish optical coherence. We cannot simply look at a clock ticking every $10^{-15}$ s and verify that it has a definite phase, because we cannot perceive anything in $10^{-15} \mathrm{~s}$.

My rebuttal applies not only to optical frequencies. Experiments show that our perception of time has a resolution in the range of tens or even hundreds of 
milliseconds [6]. Thus we cannot establish the absolute phase of any oscillator of frequency greater than a few tens of Hertz. At higher frequencies we can only establish the phase of one oscillator relative to another oscillator. This conclusion is not altered by oscillations obtained by frequency combs or $2^{n}$ upling [7]. The timing of the zeros of the highest harmonic can be no more accurately defined than that of the fundamental.

From personal observation, discussions between physicists about the existence of 'true coherence' or 'mean fields' often end with one party waving an arm up and down, intimating that by so moving an electric charge, a mean field would be produced. But that appeal fails precisely when the frequency is too fast for us consciously to move any part of our body at that frequency. There are many qualitative differences between the way radiation is generated, or detected, across the spectrum. But the only location for a fundamental (if fuzzy) dividing line in frequency between absolute and relative phase, or between 'true coherence' [1] and 'convenient fictions' [3] would be between oscillations we can observe directly and those we cannot. If this division is unpalatable that is because it relies upon the notion of absolute time. By abandoning this ill-conceived notion, the dividing line between supposed 'truth' and supposed 'fiction' disappears.

With no absolute time, all we can ever do is to use an agreed time standard, and measure phase relative to that. In this context, a laser beam is as good a 'clock' as any other. The electrodynamic $\mathbf{p}$ - A coupling allows, in principle, the laser clock to be synchronised with any material clock. The latter could then also be synchronised with any other clock based on any gauge boson field, through analogous coupling Hamiltonians [8]. The fungibility of their time standards is what makes all of these time-keepers equivalent, and justifies calling them 'clocks'. This is not an empty definition. An atom laser beam is not a clock in this sense. Its phase can only be defined or measured relative to another atom laser beam of the same species [9].

It might be objected that a laser beam is not a clock because it cannot establish a time standard between arbitrarily many parties. Eventually it will run out, or, if it is a continuous beam, its finite linewidth will mean that the later part of the beam has a random phase relative to the former part. This is of course true, and the fundamental limits are set by the finiteness of the excitation of the laser mode and laser gain medium 10]. This excitation may be very large (measured in units of $\hbar \omega$ ), but is not infinite. However, exactly the same criticism applies to any physical clock, even if we are not used to worrying about it for material clocks that typically have huge excitations. A similar point has been made in Ref. [11].

The above arguments lead inevitably to the conclusion that in quantum optical experiments there is no necessity to consider, even hypothetically, any time-keeper beyond the laser which serves as a phase reference. No other clock is superior in any fundamental sense. Now by definition a laser beam is perfectly coherent relative to itself (ignoring experimentally negligible phase and 
amplitude fluctuations, and transverse mode incoherence). Thus, the phase reference laser beam in the teleportation experiment is in a coherent state. There is no process that will make a coherent state in any stronger sense, and no need for for any stronger sense. There is no unmet need for optical coherence in continuous-variable quantum teleportation.

This letter would not be complete without discussing the recent papers [12, 13] by van Enk and Fuchs also commenting on Ref. [1]. These can be understood as an explicit calculation showing (part of) how a laser beam, without an absolute phase, can function as a clock; how the phase information can be distributed and how there is no harm in regarding the phase as real. This is essentially the same point originally made by Mølmer, that the laser phase is a 'convenient fiction' 3]. The analysis of van Enk and Fuchs gives a rigorous information-theoretic definition of 'convenience', in terms of the quantum de Finetti theorem [13]. However, it could be questioned whether convenience, however rigorously defined, is sufficient for a dispensation from the ban on the 'partition ensemble fallacy'.

The real lesson that should have been drawn from the analysis of RS is that quantum teleportation can be, and should have been, defined operationally, so that the reality of the laser phase would have been irrelevant. The reality of the laser phase can nevertheless be defended, as I have done, on the grounds that it is no less real than any other phase. Unfortunately, van Enk and Fuchs appear to accept the position of RS, that there is such a concept as 'true coherence', in that there is a time standard more real than that offered by the laser itself. Indeed, they say 12

However, recent developments 7 may make it possible to compare the phase of an optical light beam directly to the phase of a microwave field. Using this technique, the only further measurement required ... is a measurement of the absolute phase [my emphasis] of the microwave field, which is possible electronically. This measurement would create an optical coherent state from a standard laser source for the first time.

The implication is that an electronic measurement somehow makes the phase real, which it was not when it was an optical phase. As I have argued above, there is no reason to regard the laser phase as any less real than any other phase. There is nothing gained in, and no need for, appealing to any other clock in order to say that the laser is in a coherent state. If a standard laser source is used as the clock in an experiment then it creates an optical coherent state already.

To conclude, Rudolph and Sanders' concept of 'true coherence' requires (and van Enk and Fuchs appear to accept) the existence of an absolute time standard more real than that offered by the laser oscillation. I have argued that such a time standard is illusory. A laser is as good a clock as any other, and there is no need to look beyond current lasers to find optical coherence. For van 
Enk and Fuchs coherent states are still fictions, but their use is justified on the grounds of convenience, defined rigorously using the quantum de Finetti theorem. Rudolph and Sanders, by contrast, insist that if we cannot measure the phase of an oscillator (such as a laser) relative to their assumed absolute time, then we must assign it a mixed state, averaged over all possible phases. Their arguments, carried to their logical conclusion, would banish from our theories not only coherent states for lasers, but any time $t$ or phase $\phi$ if its implied resolution were beyond that of direct human experience. To scientists and engineers, this would be unacceptable pedantry.

\section{Acknowledgments}

I wish to acknowledge recent discussions with T. Rudolph, B. C. Sanders, C. Fuchs and S. L. Braunstein.

\section{References}

[1] Rudolph, T. and Sanders, B. C., 2001, Phys. Rev. Lett. 87, 077903.

[2] Furusawa, A. et al., 1998, Science 282, 706.

[3] Mølmer, K., 1996, Phys. Rev. A 55, 3195.

[4] Kok, P. and Braunstein, S. L., 2000, Phys. Rev. A 61, 042304.

[5] Pegg, D. T., 1991, J. Phys. A 24, 3031.

[6] Penrose, R., 1990, The Emperor's New Mind (London: Vintage), pp. 43944.

[7] Jones, S. J. et al., 2000, Science 288, 635.

[8] Ryder, L. H., 1985, Quantum Field Theory (Cambridge: Cambridge University Press), pp. 115-26.

[9] Wiseman, H. M., 1997, Phys. Rev. A 56, 2068.

[10] Wiseman, H. M., 1999, Phys. Rev. A 60, 4083.

[11] Egusquiza, I. L., Garay, L. J., and Raya, J. M., 1999, Phys. Rev. A 59, 3236.

[12] van Enk, S. J. and Fuchs, C. A., 2002, Phys. Rev. Lett. 88, 027902.

[13] van Enk, S. J. and Fuchs, C. A., 2002, Quantum Information and Computation 2, 151. 